\title{
A Comparative Survey for Computation of Cluster-Head in MANET
}

\author{
Mohd. Junedul Haque \\ College of Computers and \\ Information Technology \\ Taif University, KSA
}

\author{
Mohd Muntjir \\ College of Computers and \\ Information Technology \\ Taif University, KSA
}

\author{
Hussain Abu Sorrah \\ College of Computers and \\ Information Technology \\ Taif University, KSA
}

\begin{abstract}
A mobile ad hoc network is a collection of wireless nodes that can dynamically be set up anywhere and anytime without using any pre-existing network infrastructure. Several algorithms like Lowest ID, Least Cluster-head Change, Highest in-degree, Weighted Clustering Algorithm, IWCA, neural network based etc. have been proposed for clustering of nodes. They do not examine the combined effect of parameters like battery power, neighbours of node and mobility on cluster formation. Although these factors can be considered as inputs to a neural network, training the network and choosing the training algorithm is a computationally intensive hence time consuming step. In this research we address this issue by calculating computing a computationally un-intensive factor for deciding cluster-heads. This factor works in any environment and takes into account environmental changes, hence proving useful when nodes are added or subtracted dynamically from the ad-hoc network. This factor calculation could easily be built into software and can be deployed for cluster-head calculation in any ad-hoc environment with no underlying assumptions. Since we need fast calculations when the clusters change in the ad-hoc environment, coming up with a deciding factor which we can calculate fast and efficiently prevent connection breaks, dropped packets, and routing anomalies.
\end{abstract}

\section{INTRODUCTION}

With the exponential increase in the number of computing devices like mobile computers, net-books, PDA's, tablets, cellular phones and the increase in the need for connectivity at all times has magnified the importance of ad-hoc networks. Ad-hoc networks are short range networks which supposedly work without the presence of any central controller, access point or router, and provide connectivity through either single hops or multi-hops[1][2]. Example of ad-hoc networks is Bluetooth, infrared connections, and other short distance communication.

The big advantage of ad-hoc networks is that it operates without any extra circuitry other than the transmitting and receiving circuits. Although access points are also used in ad-hoc networks but such topologies can be considered as hybrid ad-hoc networks and not pure ones [7]. Initial clustering creates the clusters in the ad hoc network at a time when the wireless capable nodes are discovering each other and the cluster management algorithm maintains the clustered architecture by continually adapting to the changing network topology.

A. Terms

1. Cluster - It refers to a collection of nodes, grouped for the functioning of the networks

2. Master - Every cluster is characterized by a unique node called its master. It has certain extra responsibilities.

3. Bridge - Bridge is a node which belongs to more than one cluster. It thus has more than one master.
4. Slave - All the cluster nodes other than bridges and master are called slaves. Each slave has only one master. And hence belongs to only one cluster.

5. State - A node's state describes whether the node is a slave, bridge, master or none (none means the node is uninitialized, i.e. it does not belong to any cluster).

We will also refer to a node as slave, if its state is slave (similarly for bridge, master, none). In clustered network architecture, the whole network is divided into self-managed groups of nodes called clusters.

All the nodes within a cluster are at most two hops away from each other. These clusters continually adapt themselves to the changing network topology and new cluster configurations that are feasible with the current network topology, are created dynamically. Master (or Cluster head) is the node which is only one hop away from all the other nodes in the cluster, and carries certain extra responsibilities.

\section{RELATED WORKS}

Cluster based algorithms are among the most effective routing algorithms due to their scalability [1,2]. Clustering outperforms other routing algorithms in case of large networks. As all intercluster routing in such a scenario is through the cluster head, it is therefore more burdened than its members and tends to be a bottleneck in the system if not chosen appropriately.

The objective of any clustering algorithm is to partition the network into several clusters which is the focus of current literature in this area. Several algorithms have been suggested for clustering and Cluster head selection.

A number of clustering algorithms have been proposed, some very simple $[3,4,5]$ and some with a view of optimally utilizing the critical parameters $[6,7,8,9]$ of ad hoc networks. The classical problem of clustering involves choosing a particular node as the cluster-head so that it becomes the gateway to other nodes for the nodes of this cluster [3] [4] [7].

The responsibility of his node would then be to maintain routes, update routes, direct transmissions, calculate which all nodes fall in this cluster and others. This multi-level hierarchy prevents duplication of information and facilitates the scalability problem. Several algorithms like Lowest ID, LCC, Highest in-degree, WCA, neural network based etc. have been proposed for clustering of nodes but none of them take into account the environment specific dynamic nature of a heterogeneous ad-hoc network. They do not examine the combined effect of parameters like battery power, degree of node and mobility on cluster formation [7].

We try to solve this issue by computing a factor for deciding cluster-heads. This factor is independent of the underlying environment, computationally uninventive and takes into account environmental changes. 
The algorithms that are considering the different attributes in the network such as node mobility, degree of Cluster head, distance between nodes, node battery power etc. result in selecting more stable Cluster head with lesser affiliations and increased network lifetime. For networks with highly mobile nodes, mobility should be the critical parameter and for network with high traffic energy could be a critical parameter for Cluster head selection. Highly mobile nodes lead to more volatile clusters and should not be used as critical nodes. It can be concluded that the importance to the different parameters should be according to the network environment. Soft computing techniques can be applied to achieve clustering using existing algorithms or new algorithms and these techniques can lead to improved results [7].

Cluster-based control structures provide more efficient use of resources for large dynamic networks.

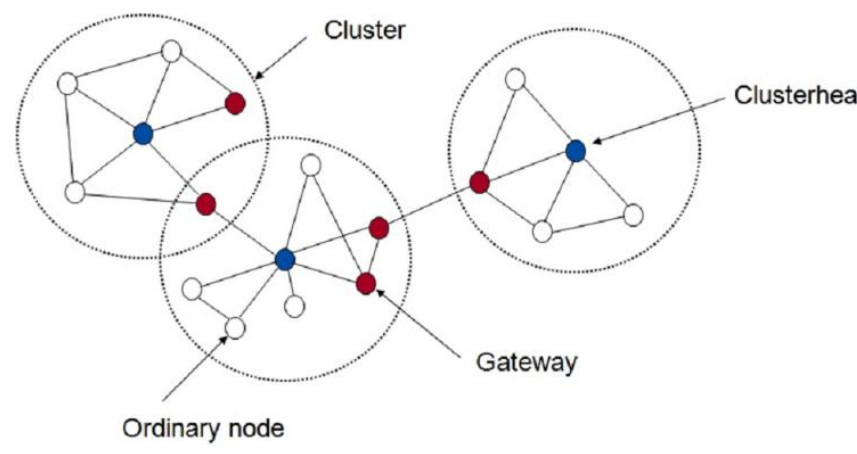

Figure 1. Link Cluster Architecture

Link-Cluster Architecture:

- Reduces interference in multiple access broadcast environment.

- Distinct clusters are formed to schedule transmissions in a contention-free way.

- Each cluster has a cluster head, one or more gateways and zero or more ordinary nodes.

- Cluster head schedules transmission and allocates resources within its cluster.

- Gateways connect adjacent clusters

Cluster heads:

- Resemble base stations in cellular networks, but dynamic

- Responsible for resource allocation

- Maintains network topology

- Acts as routers - forwards packets from one node to another

- $\quad$ Aware of its cluster members

- Aware of its one-hop neighbouring cluster heads

For clusters to communicate with each other, it is assumed that cluster heads are capable of operating in dual power mode

A cluster head uses low power mode to communicate with its immediate neighbours within its transmission range and high power mode is used for communication with neighbouring clusters Connectivity is defined as (for multiple component graph)

$$
\text { connectivity }=\frac{\text { size of largestcomponent }}{\mathrm{N}}
$$

Probability that a node is reachable from any other node ( $0-1 ; 1$ being most desirable)

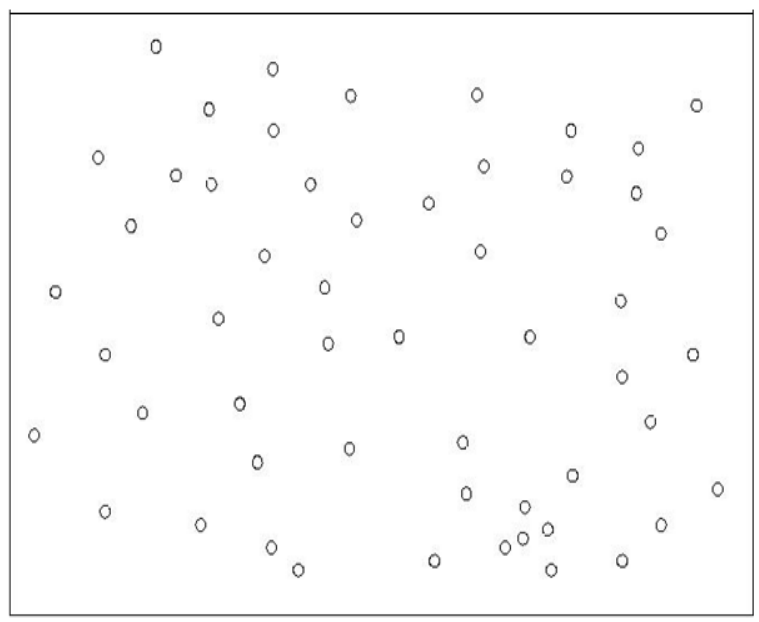

Figure 2. Network Nodes

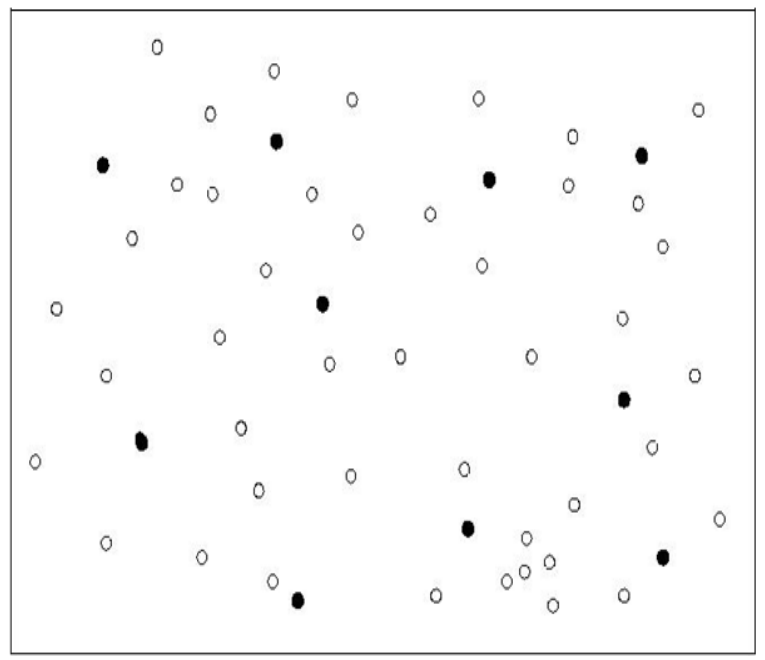

Figure 3. Identifying cluster heads

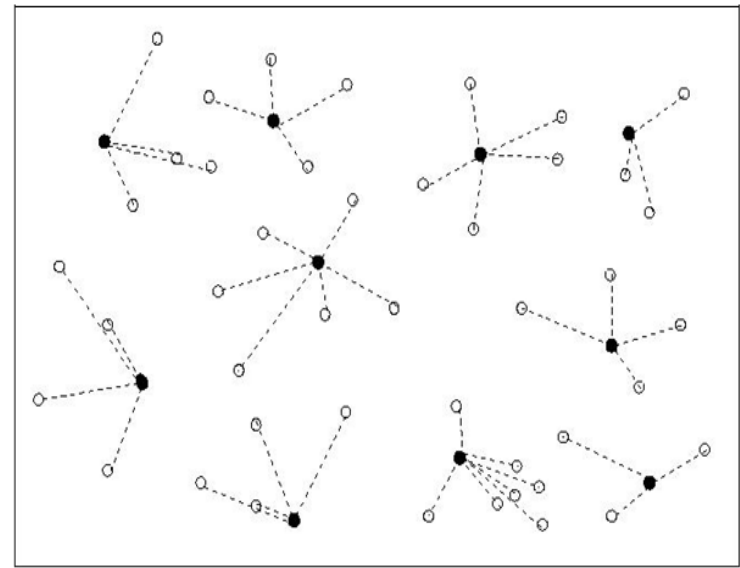

Figure 4. Forming cluster heads 


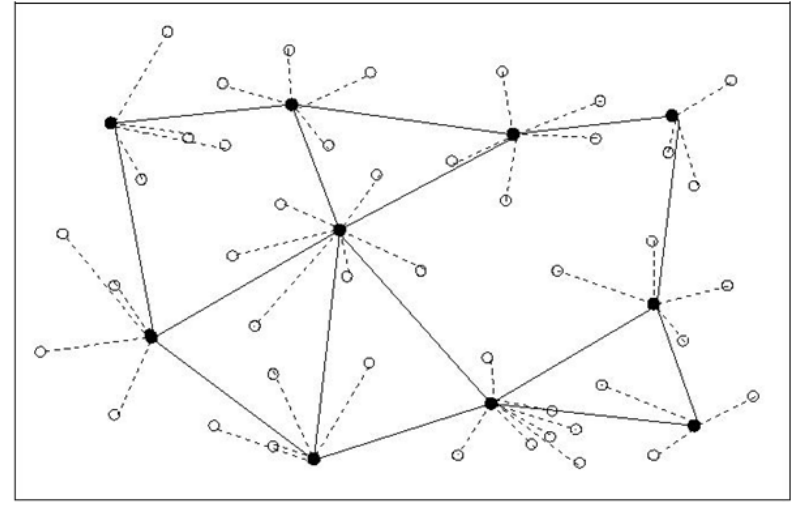

Figure 5. Finally connected clusters

\section{PROPOSED MODEL}

The need arises for a new factor calculation which takes care of the scenario described in the previous section and is independent of the underlying ad-hoc environment yet taking into account the changes occurring in it. Further its behaviors should remain and nodes changes, respectively.

We can define a new factor $F$, which will consider all the factors which are required in different cluster-head selection.

$$
\mathrm{F}=\mathrm{Bi} *\{\mathrm{SNR}(\mathrm{t}+\Delta \mathrm{t}) / \mathrm{SNR}(\mathrm{t})\} * \mathrm{Ni} / \mathfrak{£}
$$

Bi: Remaining battery power at node $\mathrm{i}$

SNR $(\mathrm{t}+\Delta \mathrm{t})$ : Signal strength at time $(\mathrm{t}+\Delta \mathrm{t})$

\section{SNR ( $\mathrm{t}$ : : Signal strength at time $\mathrm{t}$}

Ni: Number of neighbors of node $i$

$£$ : Past history of node $\mathrm{i}$

\section{OUR MOTIVATIONS}

The factor $\mathrm{F}$ that we calculate is a combination of several factors namely Battery power, signal-to- noise/signal strength at time t, signal-to-noise ratio at time $t+\Delta t$, the number of nodes which are neighbours of node $i$ and a factor which describes the past history of a node remaining the cluster-head.

A high value of $F$ would indicate a higher probability of a node being declared as a cluster-head. The theoretical correctness can be taken as follows: The battery power if becomes $0 \%$ nullifies the effect of all the other factors because the node does not function at all hence a lower battery power is not desirable for a node to qualify as a cluster- head. We measure the signal strength at two times: $t$ and $t+\Delta t$, since we want to measure the degree of movement of a nodes all around a particular node once we can take this measure for all the nodes around the ode i. A higher value is desired, as it would indicate that he node has not moved away. The direction of motion is not important as clearly the nodes are all fitted with an Omni directional transmitter and receiver.

The next factor is the number of nodes in the neighbourhood of a node $i$ and clearly indicate the approach taken by highest degree algorithm in choosing a cluster-head. Very clearly we need to choose a node as a cluster-head if the nodes that are at 1- hop distance away are high. The last factor that we can call as the trust factor stores the past history of a node being a cluster head. This factor has a value greater than 0 and less than or equal to 1 . For example if a node became a cluster head 5 times out of the 25 times it participated in cluster head election, the factor is 0.2 . But if a node never became a cluster-head then we keep a value of one for this factor, as it does not affect the factor $\mathrm{F}$ when it divides the numerator. Very clearly this factor cannot be zero and hence does not let the numerator approach infinity. Hence we prove by argument that the stated heuristic is correct to the best of our knowledge.

\section{CONCLUSION}

It is observed that for all clustering algorithms the number of clusters decrease with increase in transmission range, as more nodes are within range of other nodes for longer periods of time. Therefore, less number of clusters, which are larger in size, is formed, and mobility causes lesser number of nodes which are at the border to move in and out of range of each other. This results in decrease in the number of Cluster head changes.

We will try to calculate a new clustering factor which is computationally un-intensive, considers all factors affecting change in cluster-heads in a combined fashion and also overcomes interference anomaly by including the measurement of round trip times. One of the beauties of this factor is the simplicity in calculating the associated parameters. Since this is a newly proposed idea, no tool is directly applicable to show its implementation. So a detailed simulation study needs to be conducted to experimentally prove the validity and usefulness of the proposed clustering factor.

A comparison laid out against the already existing algorithms for calculating the cluster heads would further show its usefulness in heterogeneous environments.

\section{REFERENCES}

[1] Azzedine Boukerche "Algorithms and Protocols for Wireless and Mobile Ad Hoc Networks". Published by John Wiley \& Sons, Inc., Hoboken, New Jersey.

[2] Jun-Zhao Sun "Mobile Ad Hoc Networking: An Essential Technology for Pervasive Computing".

[3] IETF Working Group: Mobile Adhoc Networks (MANET). http://www.ietf.org/html.charters/manet-charter.html.

[4] Meeu Chawla, Jyoti Singhai, J.L.Rana "Clustering in Mobile Ad Hoc Networks: A Review”,'IJCSIS, Vol 8, No.2,2010.

[5] S.R. Das, R. Castaneda, and J. Yan, "Simulation-based performance evaluation of routing protocols for mobile ad hoc networks," Mobile Networks and Applications, 2000, 5, pp. 179-189.

[6] S.-J. Lee, M. Gerla, and C.-K. Toh, "A simulation study of tabledrive and on-demand routing protocols for mobile adhoc networks," IEEE Network, 1999, 13(4), pp. 48-54.

[7] M. Joa-Ng and I.-T. Lu, "A peer-to-peer zone-based two-level link state routing for mobile ad hoc networks,". IEEE Journal on Selected Areas in Communications, 1999, 17(8), pp. 1415-1425.

[8] L. Ji, M. Ishibashi, and M.S. Corson, "An approach to mobile ad hoc network protocol kernel design," In Proceedings of IEEE WCNC'99, New Orleans, LA, Sep. 1999, pp. 13031307.

[9] Y.-B. Ko and N. H. Vaidya, "Geocasting in mobile ad hoc netwoks: Location-based multicast algorithms,". Technical Report TR-98- 018, Texas A\&M University, Sep. 1998.

[10] M. Gerla, C.-C. Chiang, and L. Zhang, "Tree multicast strategies in mobile, multihop wireless networks," ACM/Baltzer Mobile Networks and Applications, speical issue on Mobile Ad Hoc Networking, 1999, 4(3), pp. 193207. 
[11] S. Chakrabarti and A. Mishra, "QoS issues in ad hoc wireless networks," IEEE Communications Magazine, 2001, 39(2), pp. 142-148.

[12] L. Zhou and Z. J. Haas, "Securing ad hoc networks," IEEE Network Journal, 1999, 13(6), pp. 24-30.

[13] M. Frodigh, P. Johansson, and P. Larsson. "Wireless ad hoc networking: the art of networking without a network," Ericsson Review, No.4, 2000, pp. 248-263.

[14] P. Basu, N. Khan, T. D. C. Little. "A mobility based metric for clustering in mobile ad hoc networks". Proceedings of the 21st International Conference on Distributed Computing Systems. 2001. pp. 413.

[15] D. Turgut, S.K. Das, R. Elmasri, B. Turgut. "Optimizing clustering algorithm in mobile ad hoc networks using genetic algorithmic approach". Global Telecommunications Conference, IEEE. Vol. 1. 2002. pp. 62-66.

[16] D. Turgut, B. Turgut, R. Elmasri, T. V. Le. "Optimizing clustering algorithm in mobile ad hoc networks using simulated annealing". Wireless Communications and Networking, IEEE. Vol. 3. 2003. pp.1492-1497.

[17] K. Robinson, D. Turgut, M. Chatterjee. "An entropy-based clustering in mobile ad hoc networks". Proceedings of the 2006 IEEE International Conference on Networking, Sensing and Control (ICNSC). 2006. pp. 1-5.
[18] El-Hajj, W.; Kountanis, D.; Al-Fuqaha, A.; Guizani, M. “A fuzzybased hierarchical energy efficient routing protocol for large scale mobile ad hoc networks (FEER)." IEEE International Conference on Volume 8, Issue , June 2006 Page(s):3585 - 3590 .

[19] C.-C. Chiang, H.-K. Wu, W. Liu, and M. Gerla. "Routing in clustered multihop, mobile wireless networks with fading channel.", IEEE Singapore International Conference on Networks (SICON), pages 197-211, Apr. 1997.

[20] S. Basagni. "Distributed clustering for ad hoc networks". Proc. ISPAN99 nt. Symp. On Parallel Architectures, Algorithms, and Networks. 1999. pp. 310-315.

[21] M. Chatterjee, S. K. Das, D. Turgut. "WCA: A weighted clustering algorithm for mobile ad hoc networks". Cluster Computing. Vol. 5. 2002. pp. 193-204.

[22] I. Er and W. Seah. "Mobility-based d-hop clustering algorithm for mobile ad hoc networks". IEEE Wireless Communications and Networking Conference. Vol. 4. 2004. pp. 2359-2364.

[23] P. Basu, N. Khan, T. D. C. Little. "A mobility based metric for clustering in mobile ad hoc networks". Proceedings of the 21st International Conference on Distributed Computing Systems. 2001. pp. 413. 